\title{
Physiology of heartbeat reversal in diapausing pupae of the tobacco hornworm, Manduca sexta (Lepidoptera: Sphingidae)
}

\author{
KAREL SLÁMA ${ }^{1}$ and THOMAS A. MILLER ${ }^{2}$ \\ ${ }^{1}$ Institute of Entomology, Czech Academy of Sciences, Drnovská 507, 16100 Praha 6, Czech Republic \\ ${ }^{2}$ Department of Entomology, University of California, Riverside, CA 92521, USA
}

Key words. Cardiac pulsations, extracardiac pulsations, anterograde heartbeat, retrograde heartbeat, heartbeat reversal, $\mathrm{Manduca}$ sexta

\begin{abstract}
Pulsations of dorsal vessel were monitored by the noninvasive techniques of contact thermography on the dorsal cuticle and by strain gauge detection of abdominal elongation movements. Diapausing pupae exhibited periods of forward-oriented, or anterograde pulsations (average duration of each pulsation 5-8 min, frequency of individual systolic strokes 10-15 per min) alternating with somewhat slower, backward-oriented or retrograde cardiac pulsations (average duration of each pulsation 6-10 min, frequency of systolic strokes 7-12 per $\mathrm{min}$ ). The highest rate of hemolymph flow was associated with the anterograde pulsations. We studied cardiac functions in diapausing pupae because of the almost complete absence of extracardiac hemocoelic pulsations, which are much stronger and could interfere with the recordings of heartbeat in all other developing stages. The movement of abdomen associated with the heartbeat was extremely small, only some 0.14 to $0.9 \mu \mathrm{m}$ (i.e. from one $428000^{\text {th }}$ to one $66000^{\text {th }}$ of the body length) and thus was not practical for routine recordings of heartbeat.

Simultaneous recordings from multiple thermographic sensors revealed the complete absence of retrograde cardiac pulsations in the head region. There are some indications that the retrograde pulsations were also lacking in the thoracic region of the aorta. The retrograde peristalsis appeared to be used for circulatory functions in the abdomen alone. By contrast, the anterograde cardiac pulsations underwent a profound amplification in the anterior part of the abdomen, entering thoracic aorta with considerable strength before reaching the final destination in the head region. The amplification of anterograde peristalsis was manifested by enhanced hemolymph flow towards the head associated with a two-fold increase in frequency of anterograde heartbeat before reaching the head region. The sensors distributed along the dorsal vessel revealed that the rate of the backward-oriented, retrograde cardiac flow of the hemolymph was also location specific. The rate of flow was lowest at the front of the abdomen, medium in the middle and highest close to the end of the abdomen. The finding of lowest hemolymph circulation at the beginning of the cardiac peristaltic waves suggested that the physiological "raison d' être" for heartbeat reversal was a need for differential enhancement of hemolymph flow towards the extremities of the immobile pupal body. The switchovers from the retrograde to anterograde cardiac pulsations were usually immediate, while the reciprocal, antero- to retro-switchovers were mostly associated with a brief cardiac arrest. Increasing temperature gradients (in $5^{\circ} \mathrm{C}$ steps) progressively diminished duration of both reciprocal heartbeat periods. The amplitudes of the cardiac systolic strokes also decreased with increasing temperature while the frequencies were substantially elevated.
\end{abstract}

\section{INTRODUCTION}

Heartbeat reversal in the dorsal vessel is a prominent feature of the pupal stages of all insects (Jones, 1964, 1977; McCann, 1970). The heartbeat reversal has been known for many years to consist of peristaltic waves of contraction traveling from caudal to the rostral (towards the head) direction (anterograde peristalsis) followed usually by a pause during which the heart stops, and then peristaltic waves of contraction travel in the opposite direction (retrograde peristalsis), almost always at a lower frequency of contraction (see reviews by McCann, 1970; Jones, 1977; Miller, 1985, 1997; and Wasserthal, 1996).

Patterns of heartbeat activity in pupae and adults of Lepidoptera were described in the pioneering work of Wasserthal $(1976,1980,1996)$ who perfected the thermographic recording method. This method is superior to other methods of recording heartbeat in that it is noninvasive and evokes the minimum of disturbance to the insect, and because it is an indirect measure of hemolymph flow, rather than a direct rendering of the electrical properties or movements of the dorsal vessel. Thus, ther- mographic recording can give a great deal more information about circulation than just confirming that the heart is beating (Sláma, 2000). Injury metabolism responses that accompany invasive methods of recording often obscure physiological processes. This is especially true of circulation in diapausing pupae. The non-invasive property of thermographic recording, when applied to the pupal stages, also avoids the need for immobilization, which is another abnormal procedure that can introduce abnormal responses.

It is known that the dorsal vessel organ is responsible for only a portion of the hemolymph circulation essential for healthy insects. A number of elaborate structures, collectively termed accessory pulsatile organs (Pass, 1998) ensure that appendages are perfused with hemolymph. The legs of insects are designed with bi-directional membranes and pumps to ensure flow of hemolymph all the way to the end of a given limb and back (see Miller, 1997). In addition, the coelopulse system appears to provide a different method of achieving hemolymph movement (Sláma et al., 1979). Extracardiac hemocoelic pulsations are present in the various developmental stages 
of all major groups of insects and may be responsible for hemolymph circulation through pupal appendages where cardiac pulsations have no influence and accessory pulsatile organs are lacking (Sláma, 2000).

The vital necessity of ensuring perfusion of the central nervous system of insects is also suggested by the elaborate structures and activities associated with the ventral nerve cords of many insects. Indeed, the main function of the muscular ventral diaphragm in the abdomen is almost certainly to ensure that the ventral nerve cord is thoroughly perfused with hemolymph (see Miller, 1997). It is clear that circulation is absolutely essential for healthy insects. Various elaborate structures and strategies are evolved to achieve this end, but very little is known about the details of how the central nervous system controls the function of the dorsal vessel outside of a few tantalizing suggestions and possibly three motor nerves (Davis et al., 2001; Dulcis et al., 2001).

The pupae of Manduca sexta have several pairs of large tracheal sacs, which are located between the 2nd and 6th abdominal segments. Due to voluminous resources of air within these tracheal sacs, diapausing pupae of $M$. sexta use extracardiac hemocoelic pulsations for tracheal ventilation (Sláma, 1988) only for brief 15-20min. periods of $\mathrm{CO}_{2}$ bursts occurring once in 5 to $8 \mathrm{~h}$ (Sláma, 1999). Occasional abdominal contractions or abdominal rotations serve to repair the decreasing oxygen content of tracheal sacs once every several hours.

We are investigating the in vivo function of the dorsal vessel in diapausing pupae of $M$. sexta as a prelude to studying control of heartbeat. We selected the diapause stage because there is a very regular, more or less constant heartbeat pattern and almost complete absence of the interfering extracardiac hemocoelic pulsations. The vigorous extracardiac pulsations can cause false, nonspecific thermographic responses that could be easily confused with the heartbeat (Sláma, 2000). Extracardiac hemocoelic pulsations with the frequency close to 21 beats per min occur frequently in freshly ecdysed pupae and in all nondiapause pupae. After termination of pupal diapause, they return again during the pharate adult development. In diapausing pupae of $M$. sexta, extracardiac pulsations can be temporarily evoked by mechanical disturbances or manipulations associated with attachment of thermistors to the external body wall for recordings. The pulsations always disappeared after a few min. and did not interfere with further recordings of the heartbeat. We felt it important to establish normal functions of the dorsal vessel in anticipation of more detailed work on innervation and control of heartbeat in insects, which will be described in next paper (Miller \& Sláma, 2001).

\section{MATERIAL AND METHODS}

The freshly ecdysed pupae of $M$. sexta were collected daily from stock cultures. They were maintained in darkness at room temperature. Diapausing pupae older than 3 months were stored in refrigerator at $+5^{\circ} \mathrm{C}$ before use. Sláma (2000) described contact thermographic method used for electrocardiographic recording of pupal heartbeat. These techniques were modifications of the thermographic methods applied to insects and described earlier by Wasserthal $(1976,1980)$. In this work we used low resistance semiconductor thermistors, 10NR17, manufactured by Pramet Co., Sumperk, Czech Republic (resistance $120-470 \Omega$, oval shape $400 \times 700 \mu \mathrm{m}$ ). The use of low resistance thermistors warmed slightly by an internal current is a significant change from previous methods and gives a much more sensitive detection of hemolymph movement.

The actively recording thermistor (forming a half of the resistance bridge) was positioned outside the body, usually on the midline of 4th abdominal tergite. For multisensor recordings we attached the recording thermistors on dorsal side of the head and in the middle of A1, A4 and A7 abdominal tergites. The complementary, inactive thermistor twins compensating for ambient temperature were placed on the surface of a plastic tube in the vicinity of the measured pupa. The thermistors were slightly warmed by the feeding current of the resistance bridge $(5 \mathrm{kHz}$ alternating current, options of 1, 2 or 4V, see Sláma, 2000). In principle, the movement of hemolymph under the integument disturbs the established temperature gradient created around the sensor by internal current. This produces slight cooling of the sensor, which is associated with the corresponding changes in electrical current that can be further amplified and recorded. Due to small size of the sensors, the method can reliably reproduce the frequency of pulsations up to $4 \mathrm{~Hz}$.

For the recordings we used a 4-channel measuring unit (Mikrotechna, Prague, Czech Rep.), which was especially designed for recording with strain gauges, thermistors and inductive sensors. The unit supplied stabilized $5 \mathrm{kHz}$ AC bridge current to the sensors, amplified and decoded the changes in electrical signals, and finally converted the changes into a DC output voltage that was either monitored on a PC or, alternatively, recorded by a battery of linear recorders. The advantage of using low resistance thermistors was that it enabled detection of the thermographic effect by the sensors themselves, i.e., without an external source of heat for the creation of the required temperature gradients. A further advantage of our method was that the fully synchronized 4-channel setup enabled us to record heartbeat simultaneously at 4 different positions of the dorsal vessel. The main objective of our method was absolutely noninvasive character; all sensors were placed externally outside the body.

The abdominal movements were recorded by similar techniques as described above, except that thermistors were replaced by sensitive semiconductor strain-gauge sensors manufactured by Tenzometry, Zlin, Czech Rep. (type AP-115-6-12, resistance $120 \Omega$, gauge factor 115). In this work we used special posimetric transducers specifically designed for indirect recording of changes in hemocoelic pressure from the tip of the abdomen, which were described by Sláma (1984) and by Sláma \& Neven (2001). The results reported here are based on 93 single sensor or multiple sensor thermographic recordings lasting from 6 to $42 \mathrm{hrs}, 6$ combined visual-thermographic recordings and 26 recordings of heartbeat from the tip of abdomen by means of the strain-gauge sensors.

The thermographic data are presented as relative temperature changes caused by changes in convective heat transfer by the movement of hemolymph on the side of the integument directly opposite the thermistor location (thermographic responses). The correct values have been determined from calibration curves made separately for each sensor. However, in order to comply with the standard arrangement of this type of chart, we show temperature scales with arbitrary zero at the bottom. In this case, zero flow of hemolymph corresponds to the lowest flat baseline on the record (which occurs during cardiac and extracardiac arrest), while an increased hemolymph flow (indicating greater heat transfer) was recorded as an upward deflection. There were always some variations among individual 


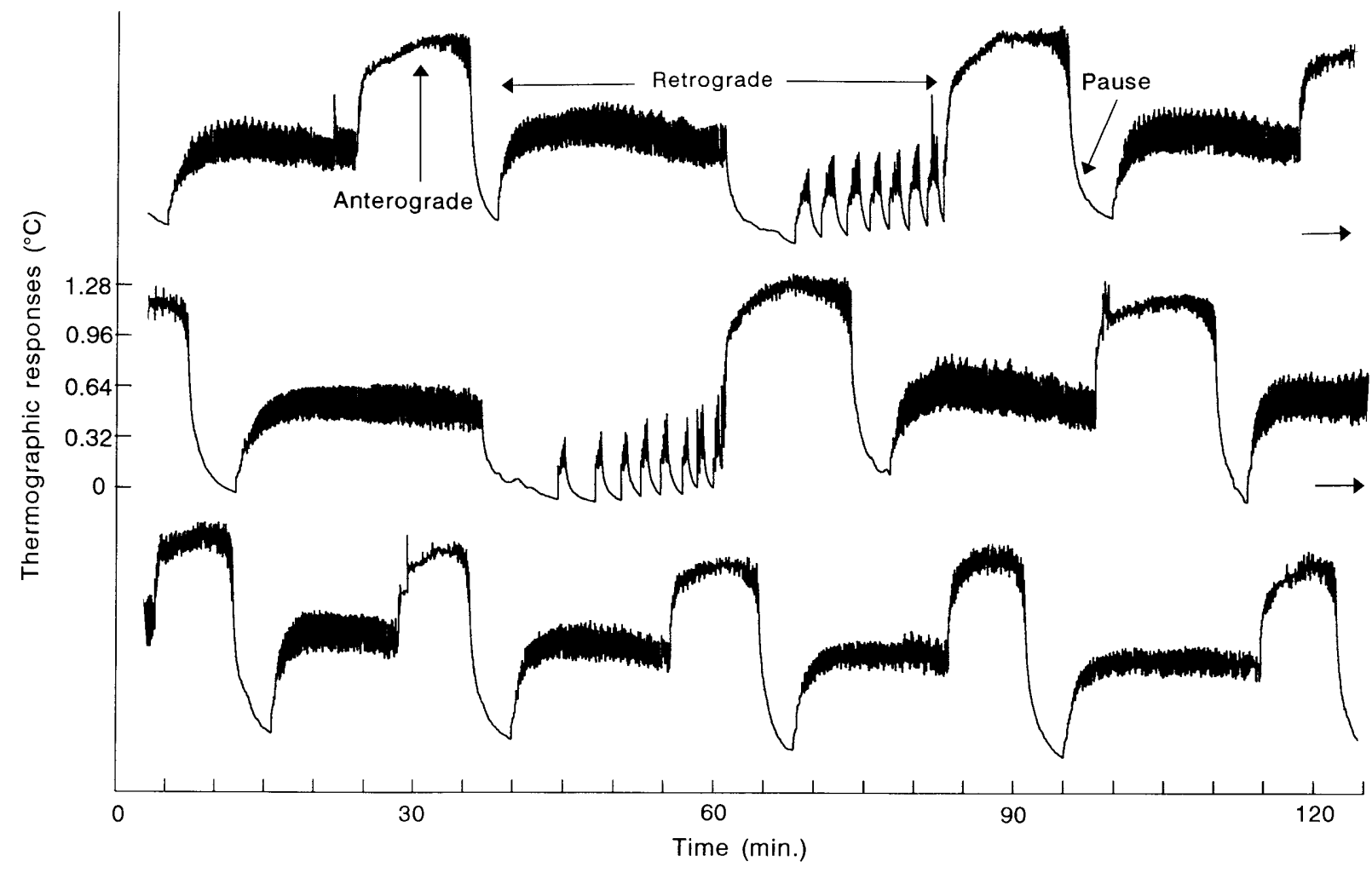

Fig. 1. Alteration of anterograde and retrograde heartbeat in non-diapausing pupa of M. sexta 6 days after the pupal ecdysis, at $23^{\circ} \mathrm{C}$. The 3 consecutive records of the cardiac activity are shown with thermographic sensor positioned in the middle of the 4 th abdominal tergite. When hemolymph flow adjacent to the thermistor increases, the cooling effect is registered as an upward deflection of the recorder pen. When the heartbeat stops and hemolymph movement ceases, the pen drops down to a relative steady state or zero flow level (indicated on the upper trace by the arrow labeled "pause").

records based primarily upon subtle differences in positioning of the sensors. This made it difficult to statistically analyze and condense the data in standard ways. We thus decided to select and show here a few individual representative records.

\section{RESULTS}

\section{Hemolymph circulation in pupae of $M$. sexta}

Fig. 1 shows a typical thermographic record of heartbeat reversal obtained from the fourth abdominal tergite area of a developing (nondiapausing) pupa in the middle of the pupal instar, at the bottom of the U-shaped curve of metabolic activity. The heartbeat pattern of this developmental stage and absence of extracardiac pulsations were quite similar with the pattern found in diapausing pupae. The periods of anterograde pulsations (arrow) were usually followed by brief resting periods indicated by the downward deflection of the thermographic recording (see pause arrow in Fig. 1). The reversed, somewhat longer, backward-oriented or retrograde pulsations of the dorsal vessel are indicated on the top trace of figure one. Note that these retrograde bouts were interrupted by a pause followed by several isolated series of retrograde pulsations.

The discontinuous series of retrograde pulsations (shown in Fig. 1) occurred especially in pupae developing without diapause or during the postdiapause adult development. The retrograde cardiac pulsations of diapausing pupae were always continuous. The most pro- nounced increase in rate of hemolymph flow through the pericardial region underlying the sensor (indicated by the pronounced upward deflection in the thermographic traces) was always associated with the anterograde heartbeat.

The record in Fig. 2 shows one complete anterograde and one retrograde bout of heartbeat in more detail. The anterograde pulsations started at a somewhat higher frequency of 15 strokes per min, and terminated at the rate of 13 strokes per min. The decreasing frequency towards the end of each bout of anterograde pulsation suggested the presence of some internal regulatory feed-back mechanism. The retrograde pulsations were usually more regular in frequency, and at a lower rate, about 8-9 strokes per min.

During and after pupal ecdysis, there were frequent alternations of the forward and backward oriented heart peristaltic contractions. At this time there were also strong extracardiac hemocoelic pulsations that occurred as often as 21 times per min. During pharate adult development the cardiac and extracardiac pulsations gradually merged into almost continuous activity as adult eclosion approached. These rather complex developmental changes in heartbeat reversal of $M$. sexta pupae will be described elsewhere. 


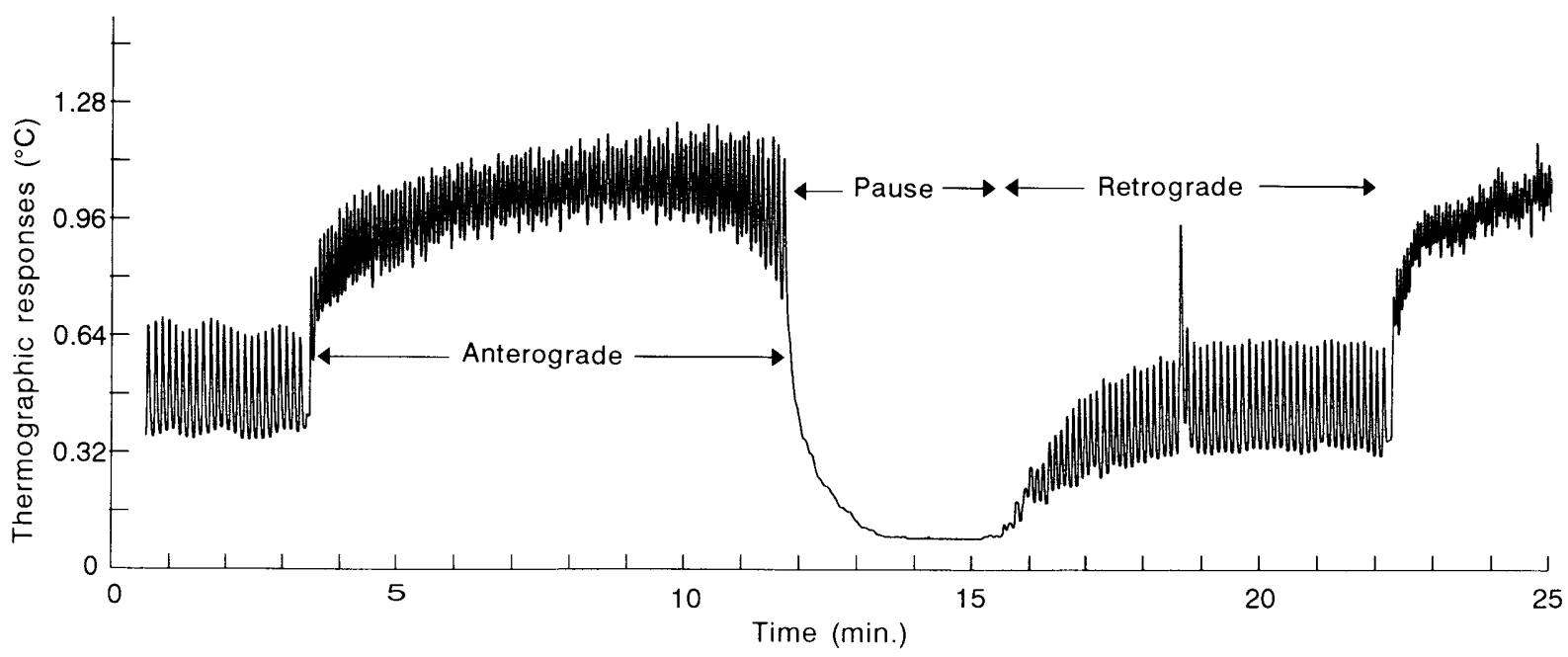

Fig. 2. Detail of a small portion from figure 1. This record shows different frequencies of the anterograde (15-13 pulses per min) and retrograde (9-8 pulses per $\mathrm{min}$ ) heartbeat. Large singular peak during the retrograde heartbeat during the 18 th min of the record is an artifact caused by a sudden abdominal contraction (confirmed visually).

\section{Passive suction inspirations}

The body segments of the pupal case are one continuous rigid structure except for flexible membranes joining the 4th through the 7 th abdominal segments, which allow movement of the abdomen. When all spiracles are tightly closed, internal (hemocoelic) pressure starts a decrease that is proportional to the rate of $\mathrm{O}_{2}$ consumption because the metabolic $\mathrm{CO}_{2}$ that is produced dissolves in carbonate buffers in the hemolymph. When a spiracular valve opens, air rushes into the spiracle with a velocity proportional to the decreased pressure (up to $1 \mathrm{kPa}$ ). The sudden inflow of air into the body results in a temporary rise in the amount of internal pressure. This can be recorded directly as changes in hemocoelic pressure or, indirectly as a prolongation of abdominal segments across the 3 elastic membranes joining them (for more details see Sláma, 1984, 1988). These respiratory patterns are known as passive suction inspirations.

The upper trace of Fig. 3 shows a strain-gauge record of changes in abdominal length, with a "saw-tooth" pattern caused by passive suction inspirations. The sharp "teeth" were produced by step-wise decreases in internal pressure (the resulting, constant, step-wise trend in abdominal contraction caused by oxygen consumption is indicated by the arrow marked A in Fig. 3). Intermittent inspirations of air through a brief opening of one of the spiracles with a periodicity of 1.3 inspirations per min. caused the sharp downward deflections, recorded as an abrupt prolongation of the abdomen. The velocity of the abdominal contraction due to $\mathrm{O}_{2}$ consumption (A) was $5.4 \mu \mathrm{m}$ per min.

The middle and lower sections in Fig. 3 show two consecutive, $22 \mathrm{~min}$. records obtained by monitoring changes in abdominal length at the time when one or more spiracular valves remained open or closed tightly only very briefly (see the "teeth" on bottom two traces in Fig. 3) during the time of these recordings. In this case the strain gauges were sensitive enough to distinguish the faster anterograde and slower retrograde activities of the dorsal vessel, both rendered as very slight movements at the tip of the abdomen. The retrograde cardiac pulsations were more distinctive as recorded at abdominal extremity; however, in contrast to the rather large pupal body size, the amplitude of movements exerted by individual strokes of the retrograde heartbeat was very small, only some 0.2 to $0.9 \mu \mathrm{m}$. The amplitude of anterograde heartbeat recorded from the tip of abdomen was smaller than 0.2 $\mu \mathrm{m}$, which was only $1 / 300000^{\text {th }}$ of the pupal body length (60 mm).

The results presented in Fig. 3 show that movements of the abdomen are very complex. In addition, periodic occurrence of large abdominal twisting movements completely overwhelmed these very sensitive force transducers and obliterated the records. As a result we abandoned the strain-gauge method and used the more reliable thermographic method for routine recordings of heartbeat in $M$. sexta.

\section{Heartbeat reversal in diapausing pupae after chilling}

Unchilled diapausing pupae of $M$. sexta have a very constant heartbeat pattern. After more than 6 months at room temperature, however, some of them spontaneously recovered from diapause and initiated adult development. Synchronized development of diapausing pupae was induced by storage of diapausing pupae at low temperatures (between $5^{\circ}$ and $10^{\circ} \mathrm{C}$ ) for a long time (one to several months). When we exposed chilled pupae to room temperature (nominally $24^{\circ} \mathrm{C}$ ) after 3 months, they initiated postdiapause adult development.

The heartbeat character and reversal pattern gradually changed as the previously chilled pupae acclimatized to room temperature over a twelve-hour period. The heartbeat activity changed again one to two weeks postchilling because the more advanced developing pharate adult stages showed quite different, postdiapause heartbeat patterns and developed strong extracardiac pulsations as well. The results obtained with these pharate 


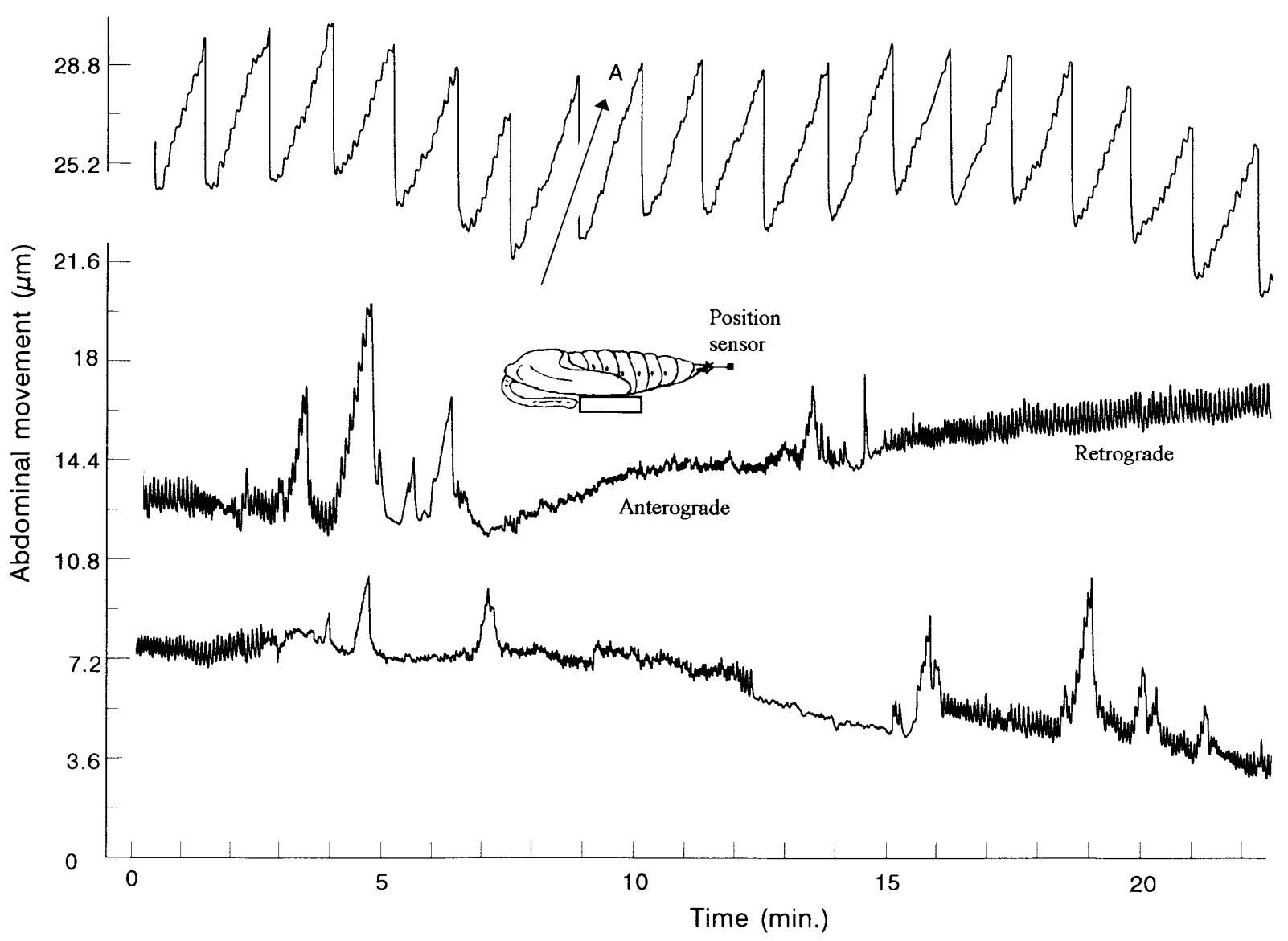

Fig. 3. A strain-gauge sensor recording the lengthening and shortening (contraction) of the abdomen in diapausing pupa of $M$. sexta at $25^{\circ} \mathrm{C}$. The inset shows the sensor attachment. Upper trace shows the saw tooth of a constant decrease in hemocoelic pressure due to steady oxygen consumption (indicated as an arrow A, the velocity of abdominal contraction was $5.4 \mu \mathrm{m}$ per min) and inspirations (rapid downward deflections at about $1.3 \mathrm{~min}$ intervals.). During the bottom two traces, certain spiracular valves remained open most time. The appearance of "teeth" in the bottom two traces (see min 5) points out to very brief periods during which spiracular valves closed tightly, giving records similar to the top trace. Note the clear record of the retrograde heartbeat superimposed on the end of the middle trace.

developmental stages have not been used here. As shown in Fig. 4, the chilled diapausing pupae needed at least 12 $\mathrm{h}$ of acclimatization at room temperature before their heartbeat patterns became more or less stabilized for the period of one or two weeks.

The higher resolution records in Fig. 5 show the results of simultaneous recording of heartbeat and abdominal movement in a previously chilled diapausing pupa, $24 \mathrm{hr}$ after transfer to room temperature. The durations of 10 min. for retrograde and $6 \mathrm{~min}$. for anterograde heartbeat were quite common among diapausing pupae of $M$. sexta, and the frequencies of both these cardiac pulsations were lower than they were in unchilled diapausing pupae. The records also demonstrated that the transition of the heartbeat reversal proceeded without pause. We can observe that the abdominal movements associated with the anterograde pulsation were very small and could barely be discerned in spite of the extremely high sensitivity of the strain-gauge transducer. The simultaneous records in Fig. 5 are important because they show that the maximum impact of heartbeat on abdominal movement (measured between min. 20 and 22 on lower trace in Fig. 5) was as low as $0.14 \mu \mathrm{m}$ and the velocity of constant abdominal contraction due to passive suction was only some $3.7 \mu \mathrm{m}$ per min.

Fig. 6 shows heartbeat reversal patterns 10 days after transfer of the chilled pupa into room temperature during the initial stages of postdiapause development. When examined in greater detail (lower trace, Fig. 6) two types of retrograde heartbeat activity were seen, one small amplitude (between min 20 and 25 of the lower trace in Fig. 6) and the other large amplitude (shown between min 7 and 10, Fig. 6, lower trace). The thermographic sensor used for the recordings in Fig. 6 was positioned on the fourth abdominal tergite. The entire anterograde peristaltic bout of activity (between minutes 10 and 16, Fig. 6 , lower trace) and the large amplitude bout of retrograde activity were both associated with significant movement of hemolymph causing the large upward displacement in the record between minutes 7 and 16 in the Fig. 6 lower trace. On the other hand, the main retrograde bout was of low amplitude with no significant effect on the thermistor indicating smaller hemolymph flow through the pericardial region near the middle of the abdomen. 


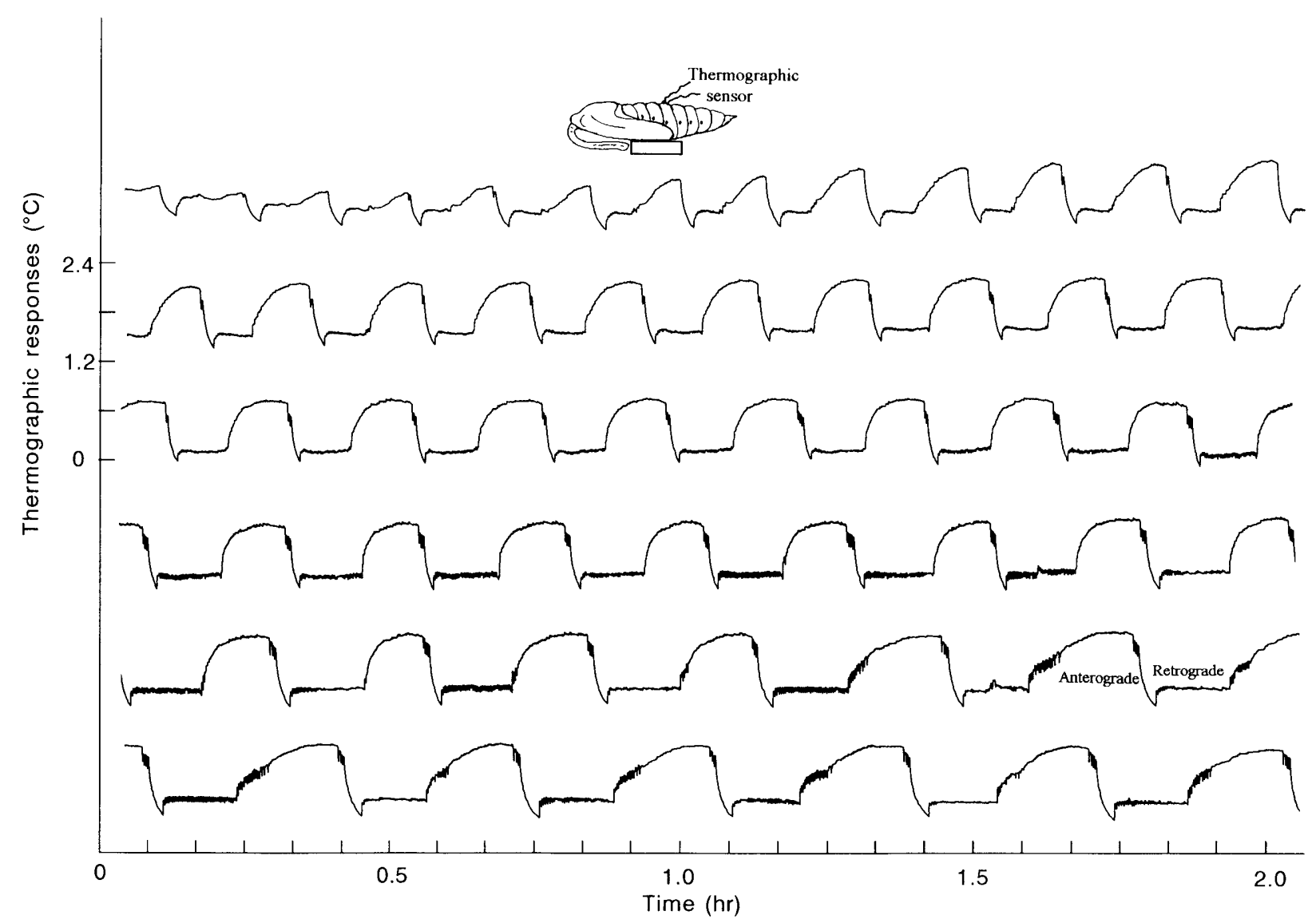

Fig. 4. Thermographic record of overall changes in the heartbeat reversal pattern during the $12 \mathrm{~h}$ period beginning by transferring a chilled, diapausing pupa from $5^{\circ} \mathrm{C}$ to $20^{\circ} \mathrm{C}$. The thermographic sensor was located on the 4 th abdominal tergite as shown on the inset.

The different character of heartbeat and distinctly different effects on hemolymph movement in the dorsal vessel in the middle of the abdomen was unexpected. It suggested that the dorsal vessel might not function uniformly along its entire length. This unusual result caused us to reexamine the heartbeat as recorded simultaneously from different parts of the dorsal vessel during ordinary cardiac reversal cycles.

\section{Differential functions of dorsal vessel in head and abdominal regions.}

The anatomy of the dorsal vessel in $M$. sexta changes drastically during development from the larvae to the adult. In the larvae the dorsal vessel lies along and is attached to the middle dorsal tergites from the tip of the abdomen to the head where it plunges underneath and ends just anterior to the brain. In the adult, the dorsal vessel in the thorax forms a convoluted path through the middle of the dorsolongitudinal flight muscles where it plays a role in removing heat from the main wing muscles during flight.

Because the dorsal vessel sinks into the thorax during development of the pupal stage, opportunities to record activity there are limited. However, it is possible to record hemolymph movement at the anterior end of the dorsal vessel where it emerges from under the brain. We recorded the heartbeat activity at the anterior end of the dorsal vessel by placing a sensor on the front of the pupae where the subintegumental stream of hemolymph is relatively high (see insets in Figs 7 and 8).

Simultaneous recordings of heartbeat from the head and fourth abdominal tergite region (Figs 7 and 8) confirmed our previous suspicions about different functions of dorsal vessel at its different parts. The most apparent difference was a complete absence of the retrograde pulsation in the head region (marked as a bar on the upper trace of Fig. 7). The sensor located on the head revealed strong hemolymph flow during the anterograde heartbeat (the larger the upward deflections in all traces of Figs 7 and 8 , the larger the hemolymph flow to carry heat away from the thermistors) while it showed a successive decline to zero hemolymph flow at the time when the abdominal sensor displayed a brief rest and switchover to the retrograde peristaltic waves (compare the upper and lower traces in Figs 7 and 8).

The results shown in Figs 7 and 8 suggested that the retrograde peristalsis of the dorsal vessel was used exclusively for abdominal circulatory functions of the heart, but not of the aorta. The clearly retrograde actions of the dorsal vessel in the abdomen and resulting significant movement of hemolymph in the dorsal vessel, appear not to include any immediate movement of hemolymph from the head region into the posterior end of the dorsal vessel 


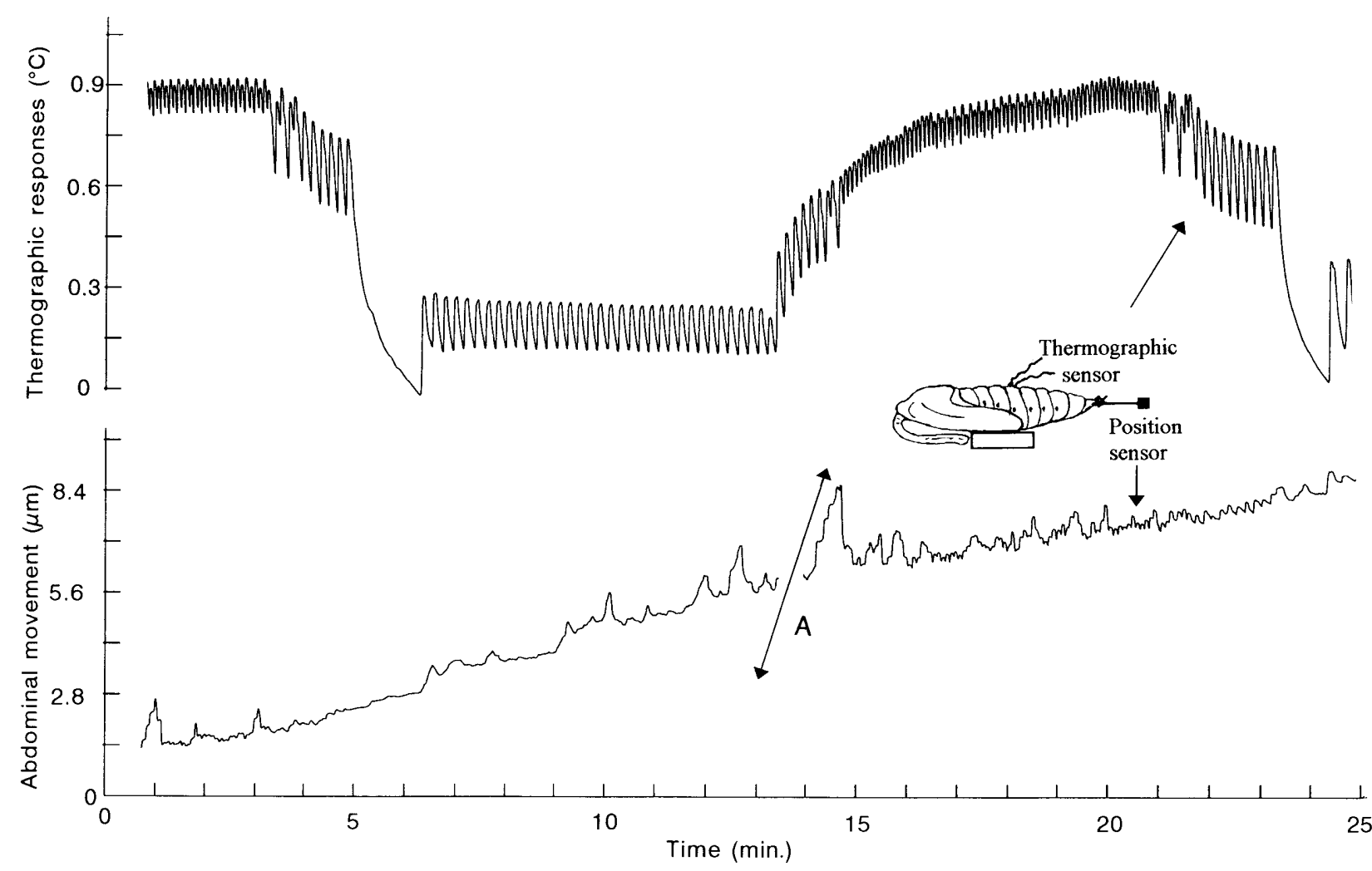

Fig. 5. The detail of heartbeat reversal taken from the thermographic preparation as shown in part in Fig. 4, after $24 \mathrm{~h}$ since translocation of the pupa from $5^{\circ} \mathrm{C}$ to $20^{\circ} \mathrm{C}$. The thermographic recording (upper trace) was realized simultaneously with recording of abdominal movement by means of the strain-gauge position sensor. The arrow line A indicates the velocity of abdominal contraction (passive suction due to $\mathrm{O}_{2}$ consumption, $3.7 \mu \mathrm{m} / \mathrm{min}$ ).

(Any possible suction effects of the retrograde abdominal heartbeat have very little, if any, effect on circulation of hemolymph through the head region). Finally, the continuous records of heartbeat in unrestrained diapausing pupa lasting two hours (Fig. 7) showed that the functions of dorsal vessel in this species and stage are very constant. We have monitored the activity of the dorsal vessel in the abdomen of Manduca sexta in some pupae for several days, weeks or even several months. In all cases, the heartbeat "reversal" is a constant feature of the activity monitored. The heartbeat patterns of different pupae had strictly individual character, there was no average pupa in this respect. Due to this, however, despite substantial accumulation of the data, conventional methods of statistical analysis did not yield practically useful results.

\section{Amplification of anterograde heartbeat between the head and abdomen}

Close examination of the details of thermographic cardiac responses in the middle of the abdomen and from the head showed that the anterograde heartbeat is two times faster in the head than it is in the abdomen (Fig. 8). There were 9 to 10 systolic anterograde contractions made by the abdominal heart per min (see upper record in Fig. 8), each of them was followed by a small, postsystolic or diastolic notch (see details in Fig. 9). The presence of such notches on the thermographic records indicated a sudden temporary arrest of hemolymph flow under the sensor.

The head region (Fig. 8, lower trace) showed 18 to 20 anterograde pulsations, with occasional differences between the odd and even pulses. The exact physiological mechanism that was responsible for amplification (doubling of the abdominal heartbeat frequency) of anterograde heartbeat before it reached the head is still unknown. We concluded that the selective advantage of this circulatory adaptation was an increased hemolymph flow through the head and thoracic region in the pupae. The amplified anterograde heartbeat may also compensate for complete absence of the retrograde pulsation in the head.

\section{Propagation of the peristaltic waves}

The sensors used for simultaneous recording of cardiac pulsations in head and abdomen (see Figs 7 and 8) were $35 \mathrm{~mm}$ apart. The anterograde peristaltic waves were observed to travel at about $60 \mathrm{~mm} / \mathrm{sec}$, at $24^{\circ} \mathrm{C}$. A wave of contraction starting at the tip of abdomen reached the head after about $1 \mathrm{sec}$; a wave starting between $3 \mathrm{~A}$ and $4 \mathrm{~A}$ abdominal segments reached the head after about 0.5 sec.

Visual observations were important in this case to confirm the unusual odd and even pattern of recorded activity in the head region. In other words, it was needed to distinguish the peristaltic waves starting at the end of 


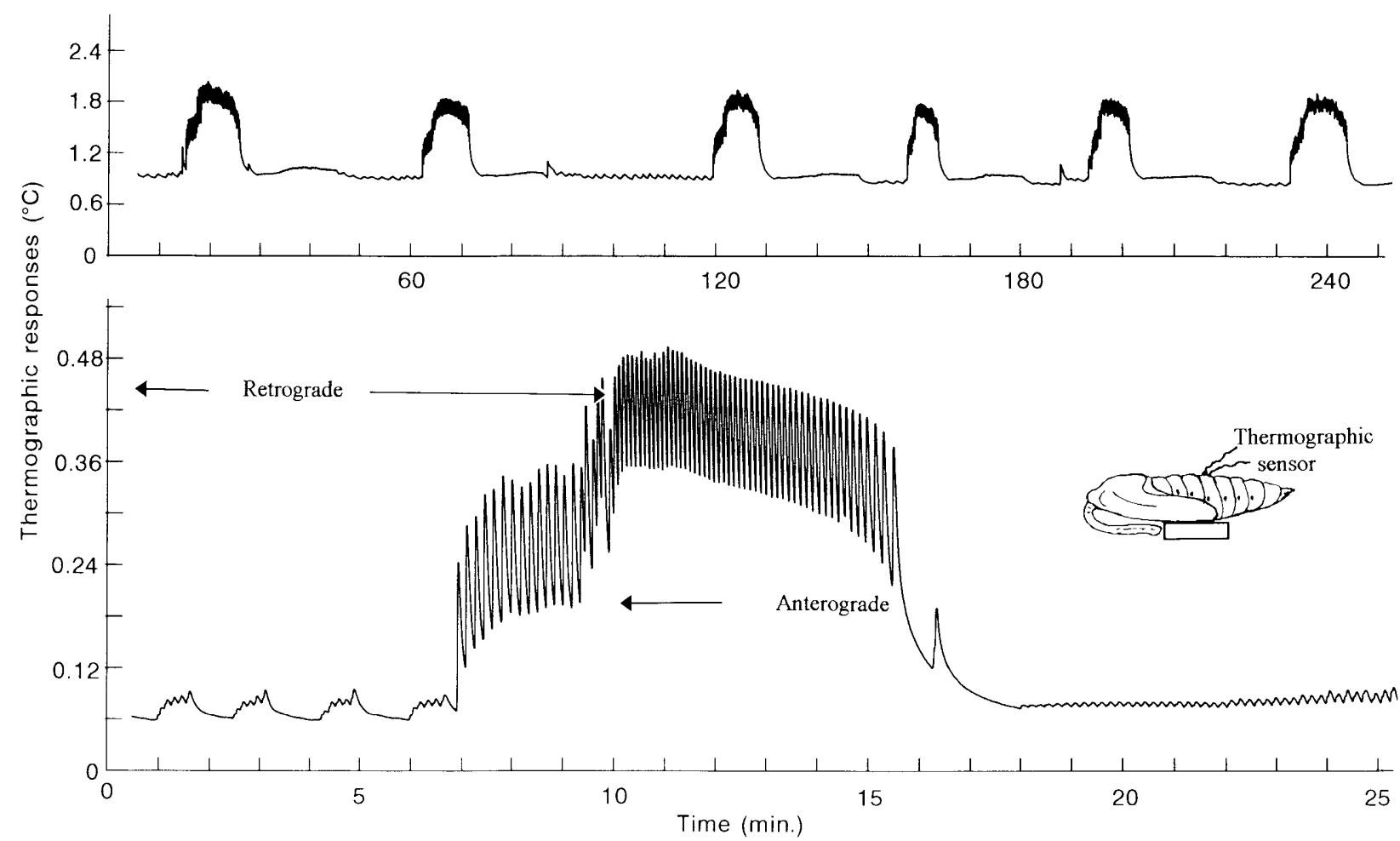

Fig. 6. The pattern of heartbeat reversal after 10 days of incubation of the chilled diapausing pupa at $20^{\circ} \mathrm{C}$. Upper record shows 10 min bouts of increased cardiac activity with increased hemolymph flow separated with approximately 25 min periods of slow retrograde heartbeat. Lower record shows the detail of one bout of cardiac activity with two kinds of retrograde and anterograde pulsations. Inset shows placement of the sensor.

abdomen from the intercalated ones originating somewhere in the proximal abdominal region. The highresolution recording in Fig. 9 illustrates the difficulty in making clear distinctions between the two types of anterograde pulses of different origin. There were 8 pulses per min recorded by the sensor on the fourth abdominal segment, while there were 8 smaller and 8 larger pulses of hemolymph flow per min in the head. After careful chronological analysis of the two records in Fig. 9 (including records with expanded time scale which are not shown here), we have determined that the larger, more efficient pulses in the head region corresponded to the intercalated peristaltic waves originating somewhere in proximal region of the abdomen. The advantage of the biphasic performance of the heart seemed obvious. It substantially enhanced the rate of hemolymph circulation through the proximal (anterior) part of the pupal body.

In addition, a new anterior wave was initiated at the end of the abdomen and was sent forward long before the previous one has reached its destination in the head. This seemed to make special sense in large insects with long dorsal vessels. At room temperature, the velocity of propagation of the anterior peristaltic waves was close to $1 \mathrm{sec}$ from tail to the head, or $0.5 \mathrm{sec}$ from tail to the middle of the abdomen at 4th segment. It is increased in proportion to the increased frequency of the pulsations at the elevated temperatures (see below). However, the differences in propagation between the antero- and retrowaves are not directly proportional to the differences in their frequencies. In spite of their smaller frequency, the velocity of propagation of the retrograde pulses occurs to be almost identical with the faster anterograde pulses.

Under certain experimental conditions, such as for instance after mechanical disturbance, manipulations or after increased temperature, the anterograde pulsations recorded from 2nd to 4th abdominal tergite did not show only one oscillation, but showed two or three systolic notches intercalated among the main peristaltic waves coming from the tail (see Fig. 9, between 180 and 240 sec, and the inset in Fig. 9). A reasonable explanation for these vibration movements of the heart is still lacking. In contrast to this, the retrograde, purely abdominal heartbeat exhibited in most cases simple, regular peaks without further oscillations in the form of systolic notches as described above for the reciprocal pulse (see Fig. 2 for comparison).

\section{Details of abdominal function of dorsal vessel in $M$. sexta pupae}

The recording of heartbeat from the terminal abdominal segments is a real experimental challenge. The pupal abdominal tip performs periodically large twisting movements or complete rotations, which can easily destroy delicate electronic sensors. We managed to solve this problem by connecting the sensors by long, thin copper wires, which moved around with the abdomen and prevented destruction of the sensors. In this way we obtained simultaneous recordings of the heartbeat from both 


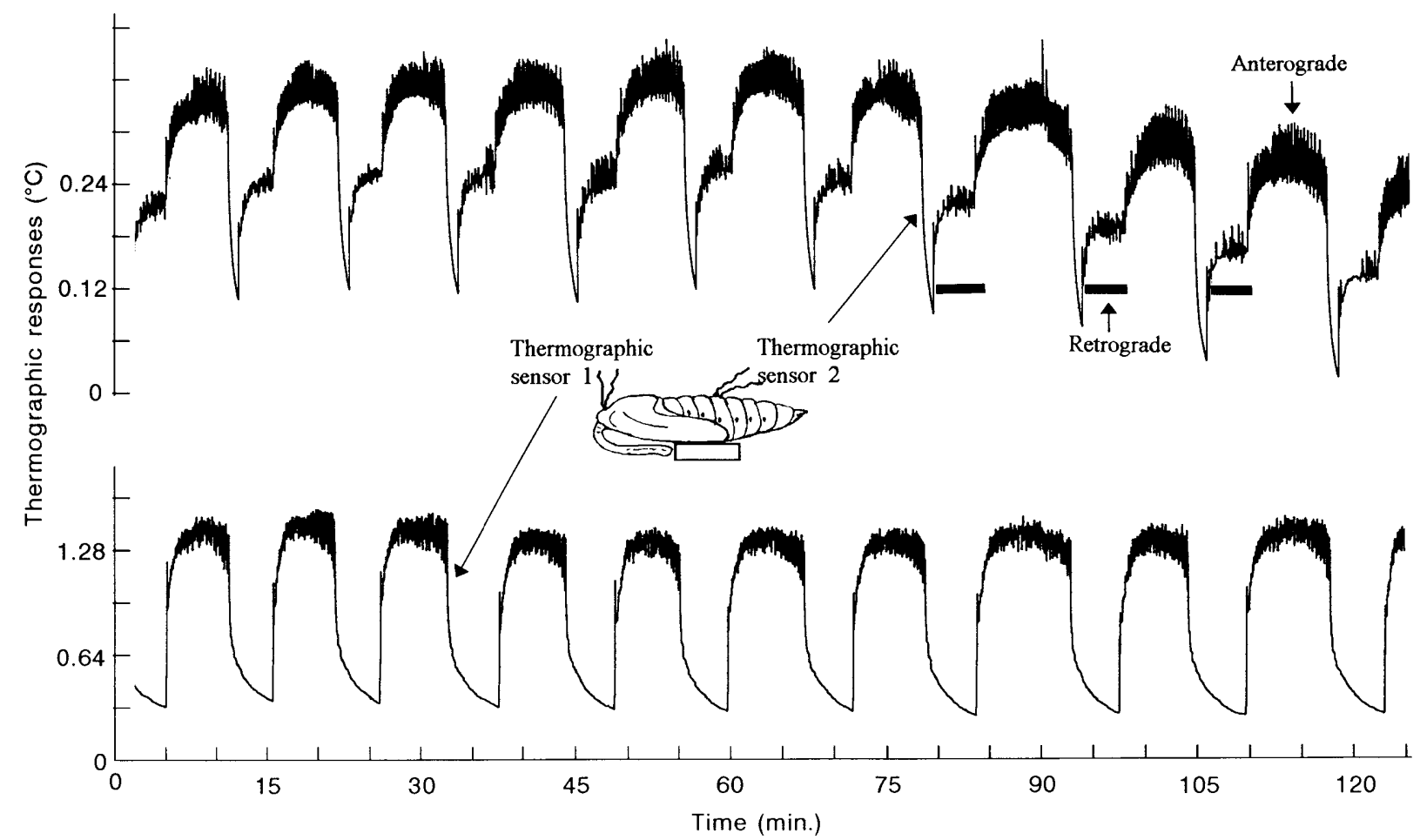

Fig. 7. Synchronized recordings from unchilled diapausing pupa at $24^{\circ} \mathrm{C}$ with two thermographic sensors, one of them positioned at anterior extremity of the body near the head region and the second located $35 \mathrm{~mm}$ apart, in the middle of the body on 4 th abdominal tergite (see inset drawing). Note that the anterior sensor exhibits selectively only the anterograde but not the retrograde peristaltic cardiac pulsations.

extremities and also from the middle of the pupal body. This type of complex multisensor recording has never been realized, so far as we know. The traces in Fig. 10 and 11 were more or less characteristic for all these multisensor recordings we obtained.

The overall patterns of dorsal vessel functions at different parts of the body, as shown by high sensitivity recordings in Fig. 10 and 11, sheds new light on normal cardiac function. Keeping in mind that an increase in hemolymph flow is indicated by an upward deflection of the recorder pen and a downward trace indicates decreasing flow, Fig. 10 shows that the entire dorsal vessel stops beating for a very brief period immediately after a vigorous bout of anterograde activity. During anterograde pulsations, the intensity of the associated hemolymph flow increased successively towards the front end of the body (indicated by the amount of upward deflection in Figs 10 and 11). During bouts of retrograde pulsations, no hemolymph flow was recorded from the sensor on the head, a medium flow and amplitude were recorded in the middle of the abdomen and a maximum flow and amplitude were recorded at the caudal end of the abdomen. The results in Figs 7-11 suggested that activity of the dorsal vessel during heartbeat reversal results in an enhanced flow of hemolymph towards both ends of the body.

During anterograde bouts of activity (Fig. 11), large amounts of hemolymph flowed past the sensor located on the head; however, individual pulsations of heartbeat activity were not recorded. This may be associated with the fact that the sensor situated externally on the head was not close to the pulsating organ (aorta). The comparatively small amplitude of heartbeat on thermographic records is also a common feature of the pharate adult stages, when dorsal vessel becomes separated from the outside sensor by newly formed epidermal layers. Finally, the data in Fig. 10 and 11 show that the switchover from retrograde to anterograde pulsation is almost immediate, without interruption. By contrast, the switchover from anterograde to retrograde pulsation is separated in most cases, but not always, by a short period of cardiac arrest. In addition, the beginning of retrograde pulsations was preceded in Fig. 10 and 11 by a single, very large, individual contraction. Curiously enough, this isolated contraction of the heart, which preceded retrograde pulsations does not originate at the beginning of abdomen, but paradoxically, came from the extreme terminal end of the abdominal part of the dorsal vessel. It seemed counter-intuitive that peristaltic pulsations should start at the end of the organ towards which the peristaltic waves of contraction were moving.

We examined the activity of the dorsal vessel in the abdomen more closely by placing three thermographic thermistors along the dorsal midline of the abdomen (Fig. 12, see inset figure for electrode placement). It was clearer from these records that indeed retrograde activity was being initiated at the terminal segment of the dorsal vessel. Note, for example, the conspicuous single pulse of 


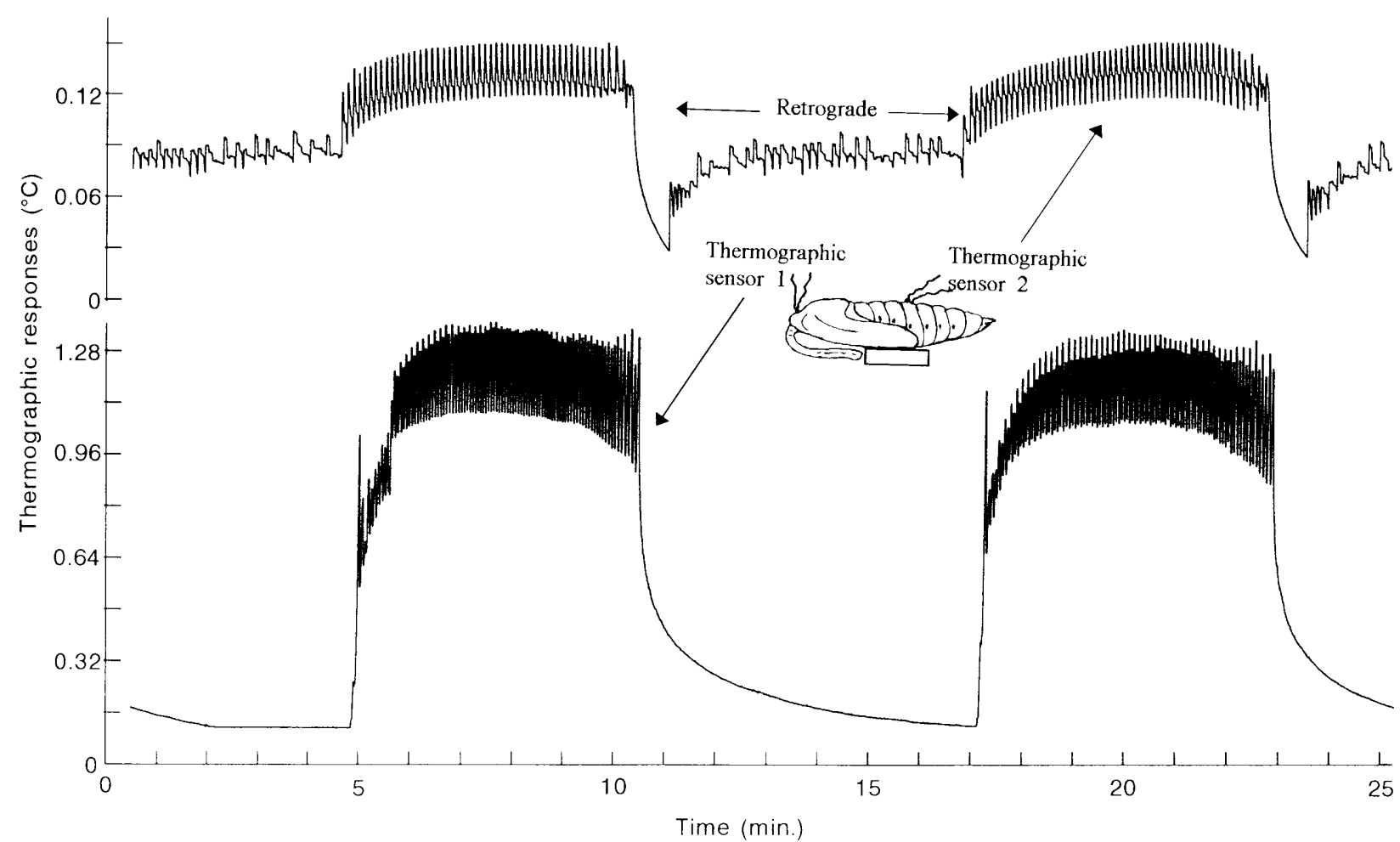

Fig. 8. A detail from Fig. 7 showing two anterograde heartbeat bouts with expanded sensitivity and time scales. We can observe a relatively large hemolymph flow through the head region during anterograde cardiac pulsation. The frequency of anterograde systolic cardiac contractions was doubled before reaching the head region. The retrograde cardiac pulses did not occur in the head region, which is indicated by the drop of hemolymph flow to zero (see min 11-17 on the bottom trace).

hemolymph flow at minute 7 of the bottom trace. The pulse slightly precedes spikes in the middle and top traces that were recorded at the more anterior parts of the dorsal vessel in the abdomen.

We measured a progressively larger amplitude of hemolymph pulses (heartbeat amplitude) from anterior electrode to the most posterior electrode (Fig. 12). The amplitude of anterograde pulsations of the entire dorsal vessel in the abdomen was muted, but hemolymph flow was significant as indicated by the upward deflection of all three traces in the anterograde period. However, there was a substantial increase of the rate of hemolymph flow going from the tip ( 8 th segment) towards the middle (4th segment) of the abdomen, but the record from the front of the abdomen (1st segment) showed a significantly lower amount of comparative hemolymph flow.

\section{Increasing temperature shortened the duration of reversal bouts of heartbeat}

It is a common feature of most neuromuscular contractions that they increase in frequency and decrease in the amplitude in response to rising temperature. Fig. 13 illustrates this general thermodynamic rule in the form of several records obtained with a single diapausing pupa of $M$. sexta. The recordings started at $20^{\circ} \mathrm{C}$ and the temperature was raised up in $5^{\circ} \mathrm{C}$ steps. When decreasing the temperature through the same steps, starting at $35^{\circ} \mathrm{C}$, there were slightly different relationships with respect to decreasing frequency of the pulsations, however, in general the results were basically similar with those shown in Fig. 13.
The rate of the heartbeat was affected by altered temperature surprisingly quickly, because the essential transition period between the two temperature patterns lasted actually only a few min.. We assume, therefore, that the refractory period depended only on the period of temperature equilibration within the pupal body. However, stabilization of the reversal and accommodation of the specific heartbeat pattern to the altered temperature required longer periods, 2 to 3 hrs. For this reason, the sample records in Fig. 13 have been taken from the long, continuous records after 2 hrs allowance for temperature equilibration.

The diapausing pupae of $M$. sexta are adapted in their natural environment to overwintering in the soil. The soil temperature changes rather slowly, the most common temperature ranges are between 5 and $10^{\circ} \mathrm{C}$. This indicates that exposures of diapausing pupae to room temperatures above $25^{\circ} \mathrm{C}$ represent a more or less unnatural environmental condition. In order to obtain more information concerning the effects of temperature on heartbeat, we performed additional individual measurements on 3 diapausing pupae exposed to temperatures from 10 to $35^{\circ} \mathrm{C}$ (see Fig. 14, left panel). These measurements revealed that the stepwise increase of ambient temperature produced a regular and proportional decrease of durations of both the retrograde and anterograde cardiac pulsations. The right panel in Fig.14 shows, conversely, that increasing temperature gradients caused a regular increase in the heartbeat frequency (number of systolic contractions at the level of $4 \mathrm{~A}$ tergite per min., averages 

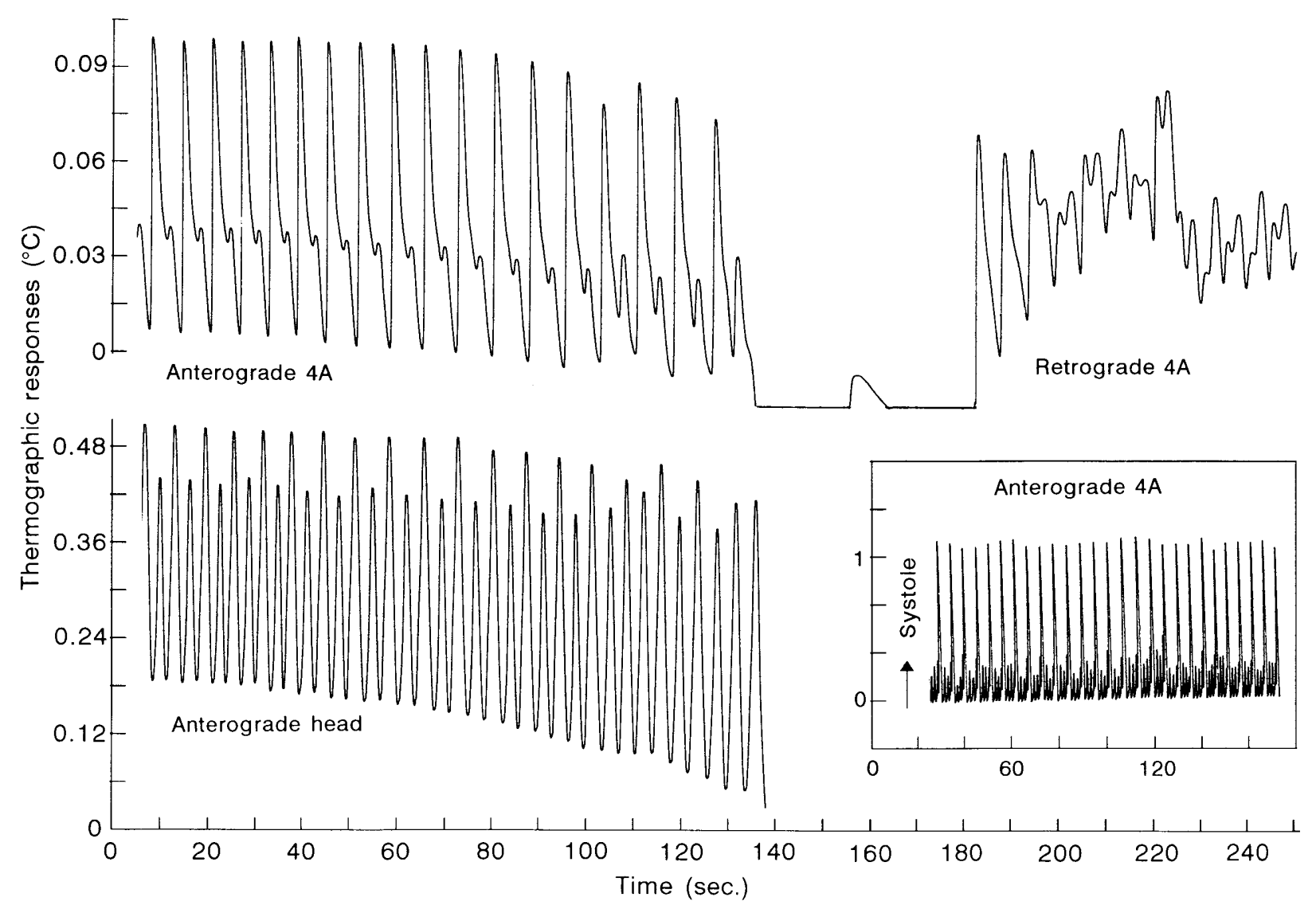

Fig. 9. High resolution detail from Fig. 7 which shows transformation of the alternating even-odd rhythmic pattern from 4A segment into a more or less homogeneous systolic cardiac pulses at the head region. With respect to retardation of the peristaltic wave in the course of traversing the $35 \mathrm{~mm}$ distance between the two sensors, the small diastolic notches in $4 \mathrm{~A}$ resulted in the stronger systolic peaks after reaching the head region. Inset in the lower-right corner shows the result of simple visual-electric recording of movements of the heart at the level of $4 \mathrm{~A}$ tergite. In this case the systolic contractions (large peaks) appear visually followed by 3 rhythmic oscillations of the heart.

from 8 pupae) of the anterograde as well as the retrograde pulsations. The following $\mathrm{Q}_{10}$ values for the anterograde cardiac pulsations were calculated: 1.93 for $5-15^{\circ} \mathrm{C}, 2.1$ for $10-20^{\circ} \mathrm{C}, 1.82$ for $15-25^{\circ} \mathrm{C}, 1.58$ for $20-30^{\circ} \mathrm{C}$, and 1.69 for $25-35^{\circ} \mathrm{C}$. The $\mathrm{Q}_{10}$ index for anterograde pulsations was considerably higher at low temperatures. On the other hand, the corresponding $\mathrm{Q}_{10}$ values for the retrograde pulsations appeared to be more or less constant, e.g. $1.66,1.66,1.57,1.60$ and 1.55 , respectively.

\section{DISCUSSION}

The dorsal vessel of insects is very conspicuous in holometabolous larvae. It lies just under the (often transparent) cuticle along the dorsal midline. During the development of the adult stage in $M$. sexta, the thoracic portion of the dorsal vessel invaginates from this uniform dorsal position into the center of the dorsolongitudinal flight muscles in the middle of the thorax.

The pupal stage of all insects is reported to exhibit the phenomenon of heartbeat reversal (review by Jones, 1977) in which peristaltic waves of contraction travel first in an anterior direction, sometimes followed by a pause, then contractions appear to travel in the posterior direction and this cycle repeats itself throughout development in the pupa. We have not seen heartbeat reversal in the younger larval stages of $M$. sexta. Bouts of reversal of heartbeat first appear at the end of larval development. The exact start of reversals coincides with the larvae becoming immobile in preparation for pupal formation. In other words, the appearance of the reversals coincides with the body becoming rigid.

Despite many years of research, we have only fragmentary information about the innervation and control of the insect dorsal vessel (Wigglesworth, 1965; Jones, 1977; Miller, 1997). A number of substances have been termed cardioaccelerators, particularly neuropeptides and biogenic amines, such as octopamine (see Miller, 1998 for review) or cardioinhibitors as allatostatin peptides (Vilaplana et al., 1999). However, the role of such substances in controlling circulation has never been made clear. There is no consensus of opinion regarding the innervation of the insect dorsal vessel, and some authors even report a lack of innervation of the dorsal vessel. The only unequivocal statement in the literature regarding innervation of dorsal vessel is that of Jones (1977) who concluded that in all cases of apparent lack of innervation of the dorsal vessel, at the very least, nerves are reported supplying the alary muscles of the dorsal diaphragm.

The situation changed for the better with the work of Ai \& Kuwasawa (1995) describing two nerve elements from 


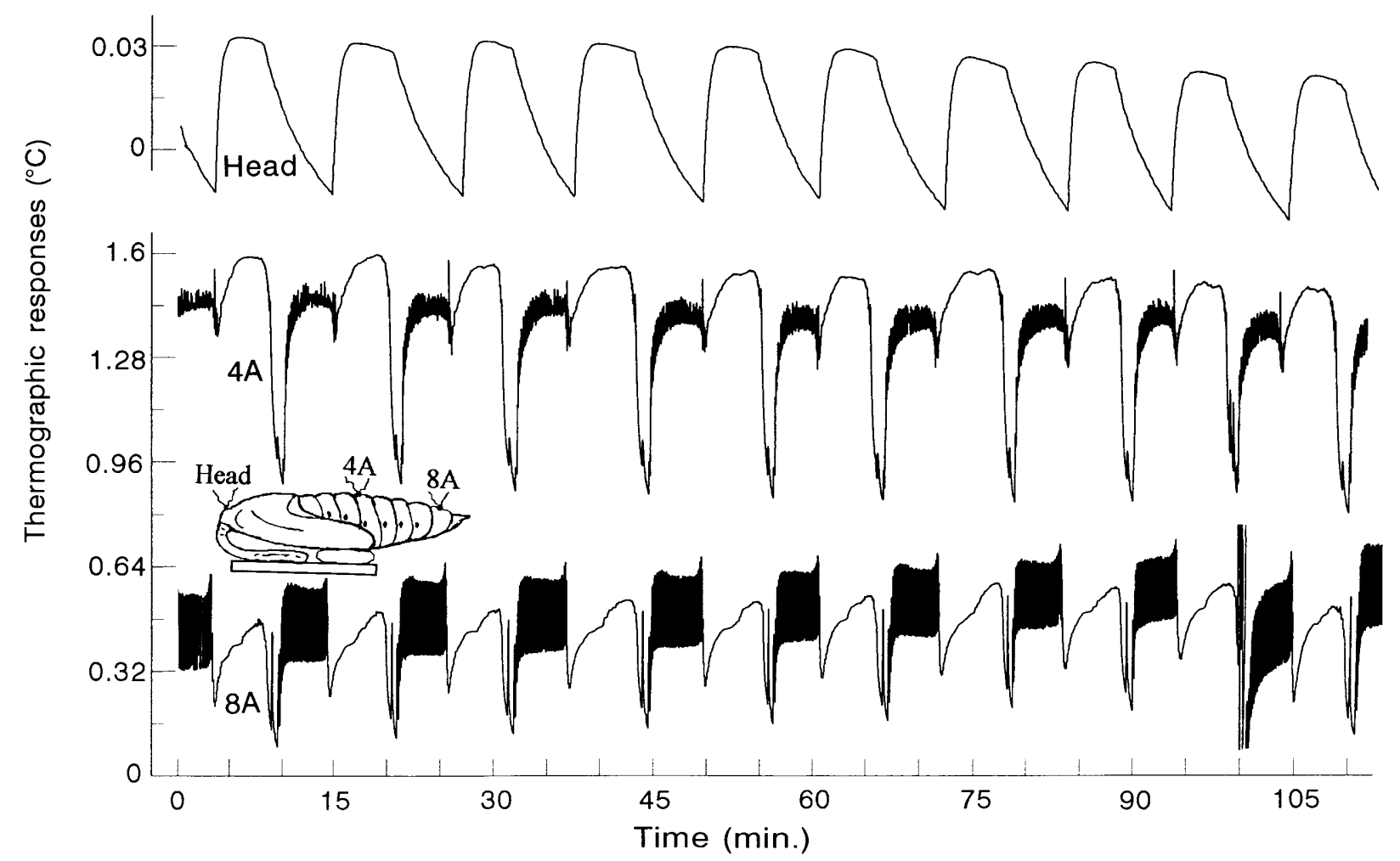

Fig. 10. The result of simultaneous thermographic recording of cardiac functions by means of 3 sensors located at either end and in the middle of the body in a young pupa determined to diapause 10 days after pupal ecdysis, stored and measured at $20^{\circ} \mathrm{C}$. The head region shows increased hemolymph flow (pen driver moving up) only during the anterograde pulsations, falling subsequently down during the pause and retrograde pulsations. By contrast, retrograde pulsations showed largest amplitude at the tip of abdomen, medium amplitude in the middle and lowest amplitude in the head region.

the recurrent nerve that, when stimulated, evoked heartbeat reversal in silkworm larvae, Bombyx mori. Not only was this the first stimulus response pathway identified in insect hearts, it was only the second and third times a cardiac motor unit was demonstrated in insects (the first was in the cockroach, Periplaneta americana, see Miller \& Usherwood, 1971). After we had initiated the present study, N. Davis and his colleagues reported a fourth motor unit, this one from the terminal abdominal ganglion in $M$. sexta that evoked heartbeat reversal as a reflex response upon stimulation (Davis et al., 2001; Dulcis et al., 2001).

Davis and his colleagues found that touching the adult body anywhere induced heartbeat reversal reactions. However, upon closer examination, he demonstrated that neurally evoked stimulation produced an immediate change from anterograde to retrograde peristalsis, but that retrograde peristalsis was insensitive to stimulation and did not evoke the opposite change from retrograde to anterograde peristalsis (N. Davis, personal communication). Neurally evoking retrograde peristalsis was also reported by Ai \& Kuwasawa (1995), but at the anterior end of Bombyx mori.

\section{Temperature effects}

Recently Smits et al. (2000) described changes in heartbeat frequency in various developmental stages of
$M$. sexta. They also reported $\mathrm{Q}_{10}$ temperature values for larval pupal and adult heart of $M$. sexta. Their data for pupae show much higher heartbeat frequency at room temperature $(21 / \mathrm{min}$.) in comparison with the data presented here. Unfortunately, they did not specify what stage of development and age of pupae they measured. The relatively low frequency of heartbeat in our experiments can be explained by the fact that we used diapause stages with the minimum metabolic intensity. The slow rate of heartbeat around 10 peristaltic waves per min. are common for immobile stages, although the lists of heartbeat rates for active stages of various insect groups show generally much higher values (Jones \& Smith, 1971). Our results on the effects of temperature on heartbeat show increasing frequency and decreasing amplitude of the cardiac pulsations. This is perhaps a reflection of a general thermodynamic rule, because in our previous paper on cockroach we obtained quite similar relationships with respect to extracardiac pulsations (Anderson et al., 1990).

\section{Cardiac-extracardiac relationships}

Using the impedance conversion technique with implanted electrodes for insect heart recording, Smits et al. (2000) found the pupal heartbeat reversal of $M$. sexta to be "unpredictable." They showed an atypical, though statistically significant pattern of activity that might indeed have resulted from the vigorous extracardiac 


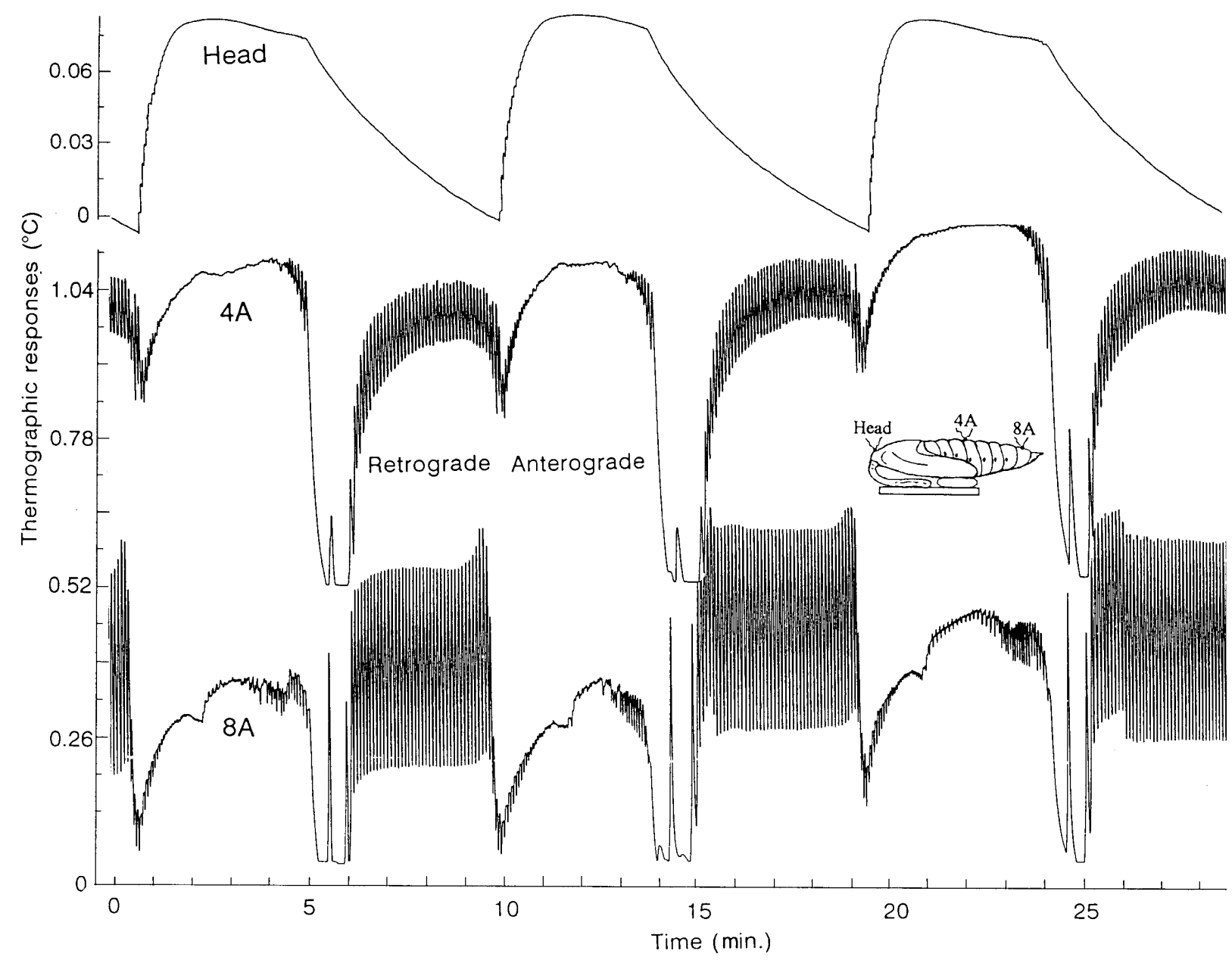

Fig. 11. A small fraction from the multisensor recording described in Fig. 10 with slightly increased sensitivity, expanded time scale and temperature increased to $25^{\circ} \mathrm{C}$. It showed that the switchover from retrograde to anterograde pulse was almost immediate whereas the anterograde-retrograde switchover occurred after a short cardiac rest. One or two very strong retrograde systolic contractions always started at the posterior end of the abdomen. The retrograde pulsation was recognized by its more or less constant frequency of 10 pulses per min, while anterograde pulsation shows frequency from 15 to 18 strokes per min.

coelopulse system superimposing movements that were assumed to be entirely due to heart beating. This combination of cardiac and extracardiac activity is very common in all developing pupae ( 21 beats per min). The impedance recording method registers movements of any kind and the exact source cannot be distinguished. However, it is known that the extracardiac pulsations can give rise to movements and pressures that are 100 to 500 times stronger than the activity associated with the dorsal vessel (Sláma, 2000).

The use of diapausing pupae, which do not exhibit extracardiac pulsations, except for a short period during the $\mathrm{CO}_{2}$ burst or after mechanical manipulation, did not allow us to determine any possible relationships between the cardiac and extracardiac pulses. This problem is more relevant to the developing pupal stages, where both these rhythmically repeated, but physiologically different, types of hemocoelic pulsations coexist together. Recent data obtained on other insect species show that internal mechanisms regulating the cardiac and extracardiac pulsations are completely different and quite independent.
Experimental confirmation of this fact has been obtained on pupae of the yellow mealworm, Tenebrio molitor (Sláma, 2000) and also on pupae of the codling moth, Cydia pomonella (Sláma \& Neven, 2001). The rhythmically repeated extracardiac pulsations often occurred in these pupae with similar but not exactly the same periodicity. Moreover, the frequency of extracardiac pulsations was usually similar with the frequency of anterograde heartbeat, which seriously increases the possibility of their mutual confusions. In both Tenebrio and Cydia, the simultaneous presence of cardiac and extracardiac pulsations has been associated with nondiapause development and with the continuous release of metabolic $\mathrm{CO}_{2}$. The diapausing pupae of $M$. sexta are of substantially larger body size, they do not exhibit regular extracardiac pulsations and their respiration is discontinuous, characterized by large bursts of $\mathrm{CO}_{2}$ in 6 to $8 \mathrm{hr}$ intervals. In spite of all of these differences, however, the basic patterns of pupal heartbeat reversal have been the same everywhere. 


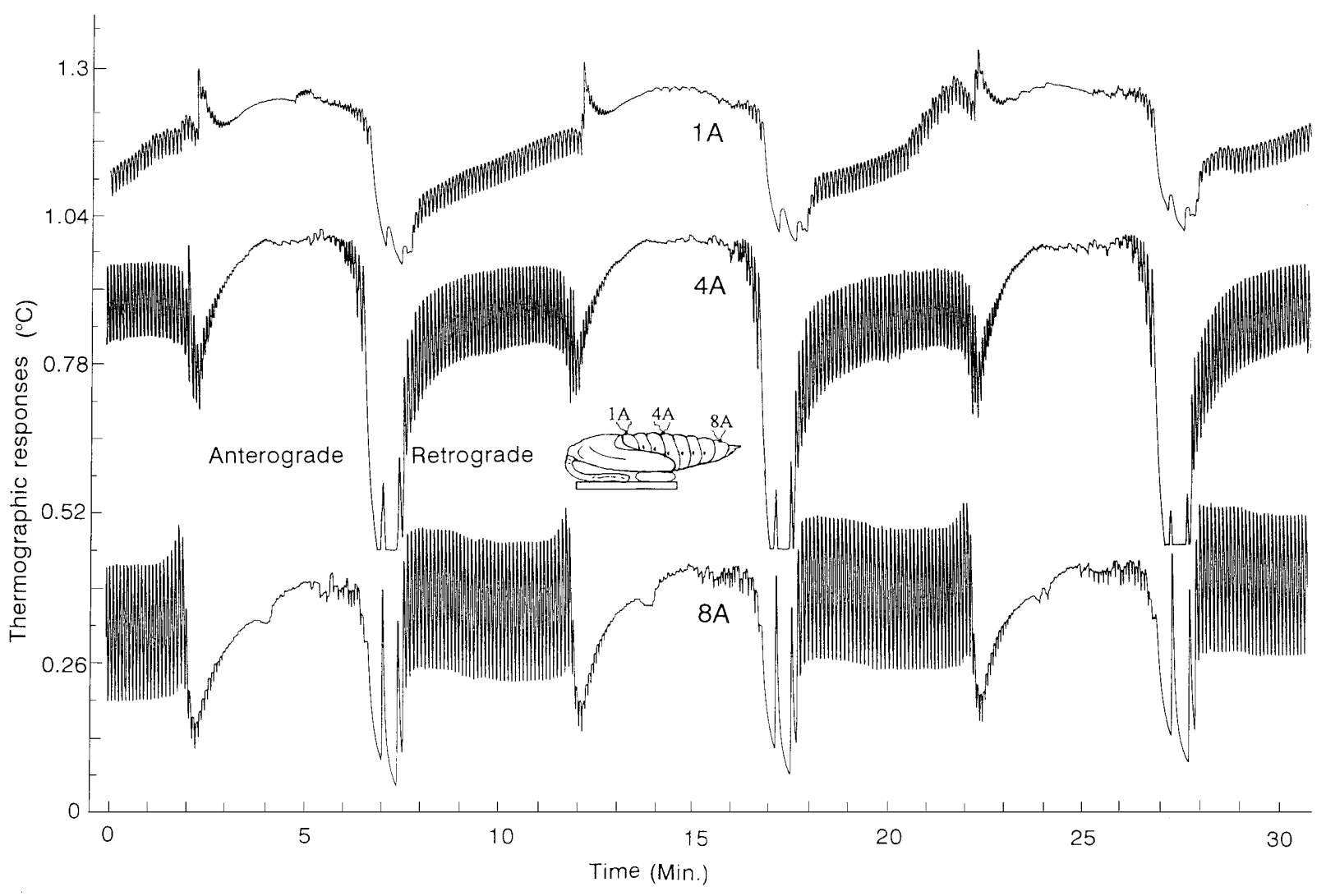

Fig. 12. Simultaneous multisensor recording of cardiac functions along the whole abdominal portion of the body in 10-day-old diapause pupa stored at $20^{\circ} \mathrm{C}$, measured at $24^{\circ} \mathrm{C}$. The amplitude of retrograde cardiac pulsations increased considerably from anterior to posterior end of the abdomen, although that of the anterograde pulsations remained more or less the same.

\section{Heartbeat on the tip of abdomen}

Our results show that the impact of heartbeat on the movement of the whole abdomen is small or negligible. This indicates that the impact of pupal heartbeat on tracheal ventilation in $M$. sexta would be also small or insignificant, which confirms previous results obtained on pupae of other insect species (Sláma, 1984, 1988, 1999; Sláma \& Neven, 2001). It is possible that the large, voluminous tracheal sacs present in the abdomen of $M$. sexta effectively buffer all small movements of the abdomen so that the impact of heartbeat on abdominal movement is here extremely small. On the other hand, the actively regulated, large abdominal contractions, twisting movements and extensive abdominal rotations, have at least a million times greater ventilatory capacity in comparison to the heartbeat.

\section{New findings obtained by using multiple electrocardiographic sensors}

The thermographic method used in this work is unique in that it not only displays the frequencies and amplitudes of the cardiac pulsations but, what is more important, it gives also reliable quantitative data on relative intensity of hemolymph flow through the pericardial sinus. Thermographic recordings from two parts of dorsal vessel were previously used by Wasserthal $(1980,1996)$ to determine the orientation and velocity of propagation of cardiac peristaltic waves. In the diapausing pupae of $M$. sexta, we have now obtained some additional data that may further extend the findings of Wasserthal.

First, we confirmed that the anterograde peristaltic waves were indeed propagated from the tip of abdomen. However, before one wave came to the proximal end of abdomen, we observed a new wave that was started and sent forward somewhere between the middle of the body and 1 st abdominal segment. In the middle of abdomen this second wave was seen only as a rudimentary wave in form of a small systolic (or diastolic?) notch. Similar notches were previously reported, but without a reasonable explanation. We determined that the second anterograde peristaltic wave, originating from the described notch, was propagated through the thoracic aorta up to the head region, where it arrived with a surge of hemolymph flow. These results showed that the performance of insect dorsal vessel was modulated at various places.

Secondly, the retrograde pulsations did not recruit hemolymph flow from the head at all, but started somewhere around the first abdominal segment (which was the first place a heartbeat could be recorded posterior to the thorax) and proceeded rearwards. A lack of hemolymph flow in the head region was recorded during the whole period of retrograde heartbeat. Based on previous literature data, we assumed that the insect dorsal vessel acted merely as a simple tube in pumping hemolymph periodically back and forth. The present results indicate that the 


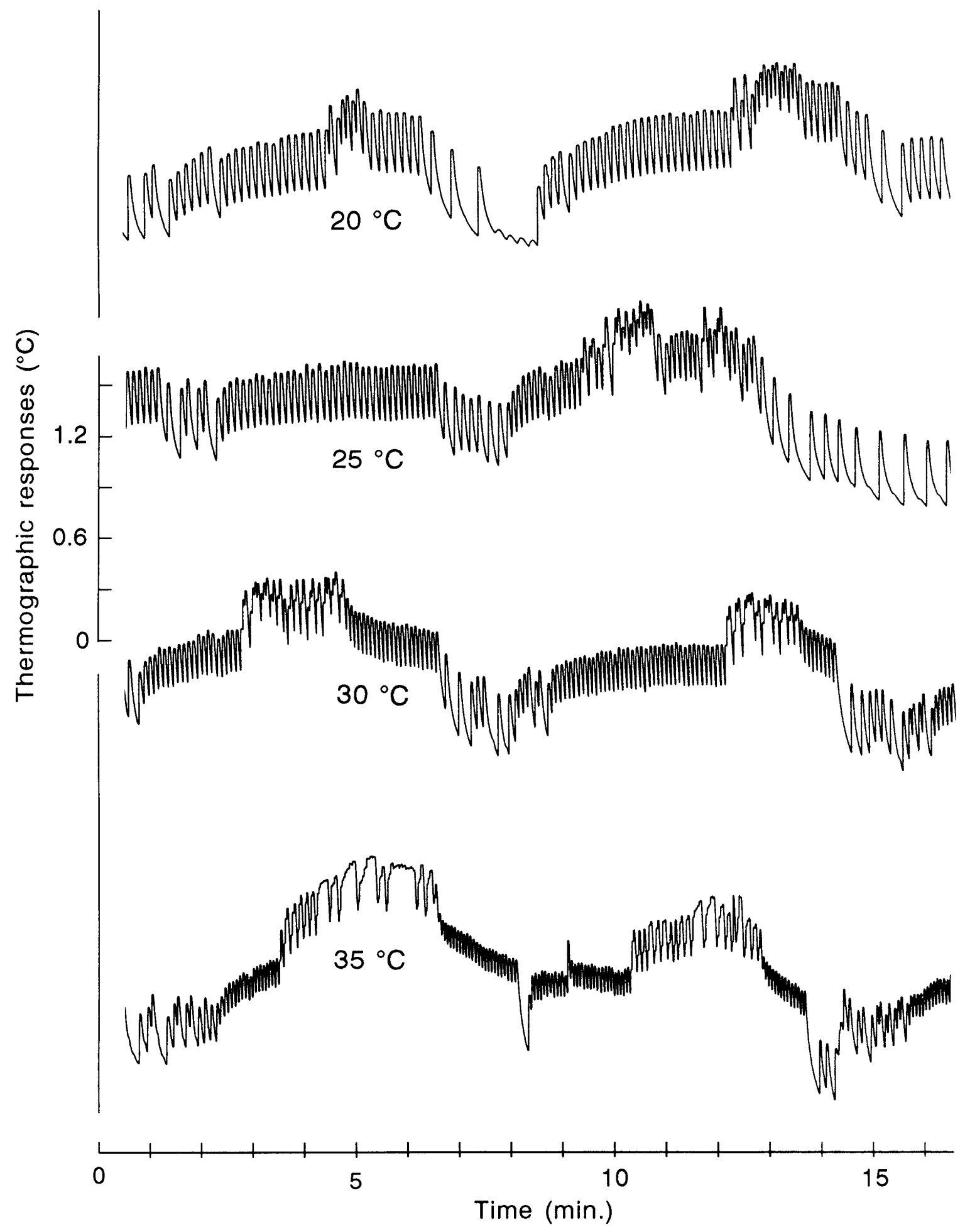

Fig. 13. Thermographic recording with the sensor located on 4A tergite showed the effect of increasing temperature on frequency and amplitude of the cardiac pulsations. The recordings were made with a chilled diapausing pupa, two days after its acclimation at $20^{\circ} \mathrm{C}$. The general phenomenon of increasing frequency and reciprocally decreasing amplitude of the pulsations was manifested very well.

circulatory functions of the dorsal vessel may be more complex than previously expected.

The third important result from simultaneous recordings came from the quantitative differences in the hemolymph flow upstream and downstream of the abdomen. A careful examination of Fig. 12 shows an increasing hemo- lymph flow towards the tip of the abdomen during retrograde peristalsis, while the expected reciprocal increase of anterograde hemolymph flow towards the base of the abdomen during anterograde peristalsis was not so pronounced. This was probably due to the retrograde pulsations terminating at and delivering hemolymph directly to 


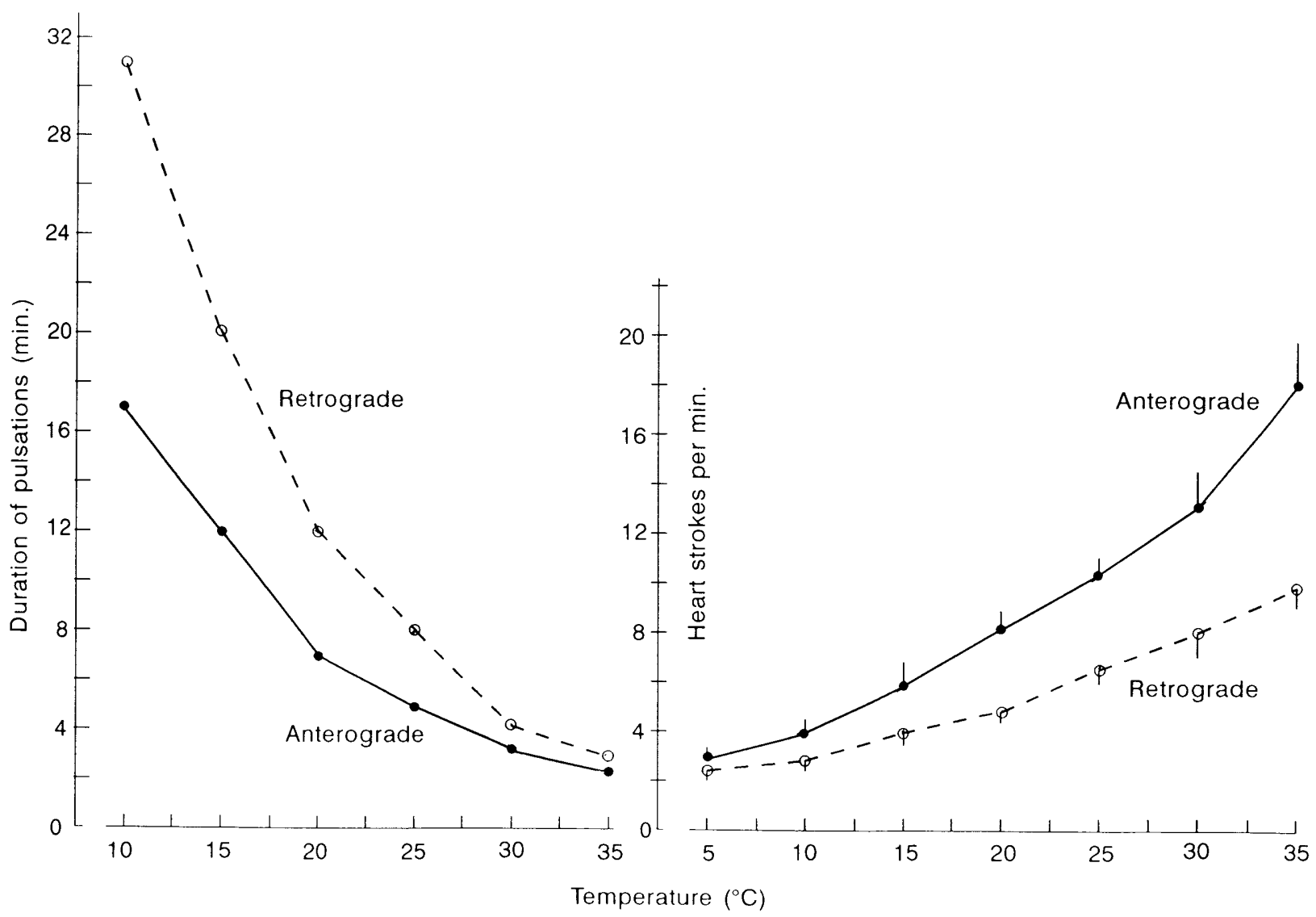

Fig. 14. The effect of increasing temperature on the duration of anterograde and retrograde cardiac pulsations (left portion, averaged from recordings on 3 individual diapausing pupae). Right portion showed the effect of increasing temperature on the frequency of anterograde and retrograde pulsations (averaged from 8 individually measured unchilled diapausing pupae; the bars represented standard error of the mean values).

the posterior abdominal extremity, whereas the destination of the anterograde hemolymph stream was not the base of the abdomen but a more distant region of the thorax and head.

The results related to differentiated hemolymph flow through different parts of the dorsal vessel can be briefly summarized so that: a) during retrograde cardiac pulsation there is minimum or no flow in the head and the amplitude of the flow increases from the front of the abdomen to the tip of the abdomen, and; b) by contrast, anterograde pulsation shows the largest amplitude of the flow and twice increased frequency of the heartbeat in the head region. From this we conclude that the main physiological reason for existence of heartbeat reversal is to selectively enhance the hemolymph flow towards both the extremities of the immobile pupal body.

\section{REFERENCES}

Ai H. \& Kuwasawa K. 1995: Neural pathways for cardiac reflexes triggered by external mechanical stimuli in larvae of Bombyx mori. J. Insect Physiol. 41: 1119-1131.

Anderson M., Sláma K. \& Miller T.A. 1990: Electrical and mechanical recording methods. In Huber, F. (ed.), Cockroaches as Models for Neurobiology: Application in Biomedical Research, 1. CRC Press, pp. 189-208.
Davis N., Dulcis D. \& Hildebrand J.G. 2001: Innervation of the heart and aorta of Manduca sexta. J. Comp. Neurol. 440: 245-260.

Dulcis D., Davis N.T. \& Hildebrand J.G. 2001: Neuronal control of heart reversal in the Hawkmoth Manduca sexta. $J$. Comp. Physiol. A 187: 837-849.

JONES J.C. 1964: The circulatory system of insects. In Rockstein, M. (ed.), The Physiology of Insecta. Academic Press, New York, San Francisco, London, pp. 2-107.

Jones J.C. 1977: The Circulatory System of Insects. Thomas Publ., Springfield, 255pp.

Jones J.C. \& SMith L.C. 1971: Heart rate; Part I. Insects. In Altman P.L. \& Ditmer D.S. (eds) Respiration and Circulation. Federation of American Societies for Experimental Biology, Bethesda MD, pp. 600-603.

Mc CanN F.V. 1970: Physiology of insect hearts. Annu. Rev. Entomol. 15: 173-200.

MiLler T.A. 1985: Structure and physiology of the circulatory system. In Kerkut G.A. \& Gilbert L.I. Compreh. Insect Physiol., Biochem., Pharmacol. 3: 289-353. Pergamon Press, Oxford, New York.

Miller T.A. 1997: Control of circulation in insects. Gen. Pharmacol. 29: 23-38.

Miller T.A. \& Sláma K. 2001: The mechanics of heartbeat reversal in diapausing pupae of Manduca sexta. $J$. Insect Physiol. (Submitted).

Miller T.A. \& Usherwood P.N.R. 1971: Studies of cardioregulation in the cockroach, Periplaneta americana. J. Exp. Biol. 54: 329-348. 
Pass G. 1998: Accessory pulsatile organs. Microscopical Anatomy of Invertebrates A11B pp. 621-640.

SLÁMA K. 1984: Recording of haemolymph pressure pulsations from the insect body surface. J. Comp. Physiol. B 154: 635-643.

SlámA K. 1988: A new look at insect respiration. Biol. Bulletin (Woods Hole) 175: 289-300.

Sláma K. 1999: Active regulation of insect respiration. Ann. Entomol. Soc. Ame. 92: 916-929

SLÁMA K. 2000: Extracardiac versus cardiac haemocoelic pulsations in pupae of the mealworm (Tenebrio molitor L.). $J$. Insect Physiol. 46: 977-992.

Sláma K., Baudry-Partiaglou N. \& Provansal-Baudez A. 1979: Control of extracardiac haemolymph pressure pulses in Tenebrio molitor. J. Insect Physiol. 25: 825-831.

SlÁmA K. \& NeVEN L. 2001: Active regulation of respiration and circulation in pupae of the codling moth (Cydia pomonella). J. Insect Physiol. 47: 1321-1336.
Smits A.W., Burggreen W.W. \& Oliveras D. 2000: Developmental changes in in vivo cardiac performance in the moth Manduca sexta. J. Exp. Biol. 203: 369-378.

Vilaplana L., Maestro J.L., Piulachs M.D. \& Bellés X. 1999: Modulation of cardiac rhythm by allatostatins in the cockroach Blatella germanica (L.) (Dictyoptera, Blatellidae). $J$. Insect Physiol. 45: 1057-1064.

WASSERTHAL L.T. 1976: Heartbeat reversal and its coordination with accessory pulsatile organs and abdominal movements in Lepidoptera. Experientia 32: 577-578.

WASSERTHAL L.T. 1980: Oscillating haemolymph "circulation" in the butterfly Papilio machaon L. revealed by contact thermography and photocell measurements. J. Comp. Physiol. 139: $145-163$.

WASSERTHAL L.T. 1996: Interaction of circulation and tracheal ventilation in holometabolous insects. Adv. Insect Physiol. 26: 297-351.

Wigglesworth V.B. 1965: The Principles of Insect Physiology. 6th ed. Methuen, London, New York, 741pp.

Received July 30, 2001; revised September 11, 2001; accepted September 21, 2001 